\title{
Fokker-Planck equation approach to the description of soliton statistics in optical fiber transmission systems
}

\author{
Stanislav A. Derevyanko \\ Photonics Research Group, Aston University, Birmingham B4 7ET, UK, and Institute for Radiophysics and \\ Electronics NASU, Kharkov 61085, Ukraine \\ Sergei K. Turitsyn \\ Photonics Research Group, Aston University, Birmingham B4 7ET, UK \\ Dennis A. Yakushev \\ Institute for Radiophysics and Electronics, National Academy of Sciences of Ukraine, Kharkov 61085, Ukraine
}

\begin{abstract}
Received September 9, 2004; revised manuscript received November 3, 2004; accepted November 19, 2004
We derive rigorously the Fokker-Planck equation that governs the statistics of soliton parameters in optical transmission lines in the presence of additive amplifier spontaneous emission. We demonstrate that these statistics are generally non-Gaussian. We present exact marginal probability-density functions for soliton parameters for some cases. A WKB approach is applied to describe the tails of the probability-density functions. (C) 2005 Optical Society of America

OCIS codes: $060.2330,190.5530$.
\end{abstract}

\section{INTRODUCTION}

Soliton transmission in optical fiber lines is a research area that belongs both to fundamental science (including such different areas as the theory of integrable systems, nonlinear optics, and statistics) and to applications. The research in this field has already had a serious effect on the design and development of practical systems that use the return-to-zero (RZ) data format. The major limitations imposed on the transmission distance in long-haul fiber lines are due to amplifier spontaneous emission (ASE), which introduces both noisy radiation and jitter into the carrier pulses and thus causes the quality of the transmission to deteriorate. In their pioneering work Gordon and Haus ${ }^{1}$ (see also Ref. 2 for the mathematical theory of the effect) studied timing jitter in soliton transmission and derived the celebrated result that the timing fluctuations along the transmission line grow as the cube of propagation distance. Later Mecozzi et al. ${ }^{3}$ showed that the use of passive filters can reduce timing jitter and thus extend the Gordon-Haus limit. Mollenauer et al. ${ }^{4}$ proposed a sliding-filters technique that permits the propagation distance to be increased even further. Nakazawa and colleagues ${ }^{5}$ successfully used amplitude modulators and passive filters to reach a distance of more than $10^{6} \mathrm{~km}$. For reviews of the early developments in soliton statistics see, for instance, Refs. 6 and 7. More recently, Mecozzi ${ }^{8}$ suggested the use of Butterworth filters to effectively suppress the background excitation in zero slots. As a result of recently renewed interest in phase shift keying systems, a series of papers dedicated to the statistics of phase jitter in soliton-dispersion phase shift keying systems was published. ${ }^{9-12}$

Note that the vast majority of the results have been obtained with the assumption (sometimes implicit) that the underlying statistics of soliton parameters are Gaussian. The first results for non-Gaussian single-soliton statistics have appeared only recently. Single-soliton statistics were studied by means of a functional integration technique ${ }^{13,14}$; this method allowed Falkovich et al. to obtain a series of asymptotes for the tails of the probabilitydensity functions (PDFs) that turned out to be nonGaussian. The authors of Ref. 15 applied the Ito method to analyze the statistics of the energy fluctuations in the nonlinear Schrödinger equation (NLSE) and showed that the energy statistics are substantially non-Gaussian. Non-Gaussian phase statistics in optical communication were also analyzed in Ref. 16 . In Refs. 17 and 18 the statistics of amplitude and phase of individual solitons were treated. In this paper we suggest an approach based on the Fokker-Planck equation (FPE) for the PDF of all four soliton parameters. This approach allows us to determine the area of applicability of classic Gordon-Haus results for the standard deviations of soliton parameters as well as to study the asymptotic behavior of the tails of the PDFs. We also discuss both the Ito and the Stratonovich approaches, considering the stochastic dynamics of the soliton parameters. The non-Gaussian statistics of the soliton parameters are a direct consequence of the multiplicative nature of noise in soliton equations. In this paper we derive a FPE for generic systems that include in- 
line filters. The FPE obtained contains exact and complete information on the evolution of the soliton statistics, and solving this equation analytically or numerically provides one with a complete or marginal PDF for soliton parameters. Some preliminary results have already been briefly reported. ${ }^{19}$ The Fokker-Planck approach has already been applied to the statistics of the NLSE soliton (by use of a truncated Langevin system for two soliton parameters). ${ }^{20}$ Here we derive the FPE that governs statistics of all four soliton parameters and that is important for applications in optical communications.

\section{MODEL}

The perturbed NLSE, in soliton units, that describes the combined action of filters and ASE has the following form $^{6,8}$ :

$$
\frac{\partial u}{\partial z}=\frac{i}{2} \frac{\partial^{2} u}{\partial t^{2}}+i|u|^{2} u+\frac{1}{2}\left(\alpha+\eta \frac{\partial^{2}}{\partial t^{2}}\right) u+\chi,
$$

where $\alpha$ is the extra gain to compensate for filter loss and $\eta$ is the filter strength (for more details about the units, see Ref. 6). ASE is accounted for as additive white Gaussian noise (AWGN) term $\chi$, with the following correlation properties:

$$
\begin{aligned}
\langle\chi(t, z)\rangle & =\left\langle\chi(t, z) \chi\left(t^{\prime}, z^{\prime}\right)\right\rangle=0, \\
\left\langle\chi(t, z) \chi^{*}\left(t^{\prime}, z^{\prime}\right)\right\rangle & =D \delta\left(z-z^{\prime}\right) \delta\left(t-t^{\prime}\right) .
\end{aligned}
$$

Both noise and filter action can be considered perturbations. Using the standard single-soliton ansatz

$$
\begin{aligned}
u_{0}(t, z)= & A(z) \operatorname{sech}\{A(z)[t-T(z)]\} \exp [-i \Omega(z) t \\
& +i \phi(z)]
\end{aligned}
$$

and soliton perturbation theory for arbitrary perturbation $R$ (see Refs. 6, 21, and 22), one can obtain the following dynamic equations for the soliton parameters:

$$
\begin{aligned}
\frac{\mathrm{d} A}{\mathrm{~d} z}= & \operatorname{Re} \int \mathrm{d} t g_{A}^{*}(t) R(t, z), \\
\frac{\mathrm{d} \phi}{\mathrm{d} z}= & \frac{1}{2}\left(A^{2}-\Omega^{2}\right) \\
& \quad+\operatorname{Re} \int \mathrm{d} t g_{\phi}^{*}(t, z) R(t, z), \\
\frac{\mathrm{d} \Omega}{\mathrm{d} z}= & \operatorname{Re} \int \mathrm{d} t g_{\Omega}^{*}(t, z) R(t, z), \\
\frac{\mathrm{d} T}{\mathrm{~d} z}= & -\Omega+\operatorname{Re} \int \mathrm{d} t g_{T}^{*}(t, z) R(t, z),
\end{aligned}
$$

where

$$
\begin{aligned}
g_{A}(t, z)= & u_{0}(t, z, A, \phi, \Omega, T), \\
g_{\phi}(t, z)= & \frac{i}{A}\{1-A t \\
& \times \tanh [A(t-T)]\} u_{0}(t, z, A, \phi, \Omega, T),
\end{aligned}
$$

$$
\begin{aligned}
g_{\Omega}(t, z)= & -i \\
& \times \tanh [A(t-T)] u_{0}(t, z, A, \phi, \Omega, T), \\
g_{T}(t, z)= & \frac{t-T}{A} u_{0}(t, z, A, \phi, \Omega, T) .
\end{aligned}
$$

In a noisy system with filtering, perturbation $R(t, z)$ has the form $R=(1 / 2)\left[\alpha+\eta\left(\partial_{t}\right)^{2}\right] u_{0}+\chi$.

\section{LANGEVIN EQUATIONS FOR SOLITON PARAMETERS: ITO AND STRATONOVICH APPROACHES}

Taking the projections of the perturbation onto the functions $g_{i}$, after some algebra we arrive at the following system of equations (see Ref. 6):

$$
\begin{aligned}
& \frac{\mathrm{d} A}{\mathrm{~d} z}=\alpha A-\eta\left(\Omega^{2}+A^{2} / 3\right) A+S_{A}(z), \\
& \frac{\mathrm{d} \phi}{\mathrm{d} z}=\frac{1}{2}\left(A^{2}-\Omega^{2}\right)+S_{\phi}(z), \\
& \frac{\mathrm{d} \Omega}{\mathrm{d} z}=-\frac{2}{3} \eta A^{2} \Omega+S_{\Omega}(z), \\
& \frac{\mathrm{d} T}{\mathrm{~d} z}=-\Omega+S_{T}(z) .
\end{aligned}
$$

$S_{i}$ are the projections of AWGN on perturbation functions (9)-(12):

$$
S_{i}(z) \equiv \operatorname{Re} \int \mathrm{d} t g_{i}^{*}(t, z) \chi(t, z) .
$$

This system can be viewed as a Langevin system with multiplicative noise ${ }^{23}$ :

$$
\begin{aligned}
\frac{\mathrm{d} q_{i}}{\mathrm{~d} z}=f_{i}(\mathbf{q})+\operatorname{Re} \int \mathrm{d} g_{i}{ }^{*}(t, \mathbf{q}) \eta(z, t), & \\
& i=A, \phi, \Omega, T .
\end{aligned}
$$

The set of Eqs. (13)-(16) [or Eq. (18) in compact notation] is a master set of equations that describe the adiabatic stochastic evolution of the four soliton parameters. Some special instances of this system were studied before, and the main results for soliton statistics (including the Gordon-Haus effect) were derived under certain assumptions. Note that, despite the perturbation theory that was used to derive Eqs. (13)-(16), these assumptions are valid not only for small propagation distances $z \ll 1$ when the soliton parameters do not change significantly from their initial values $A_{0}, \phi_{0}, \Omega_{0}$, and $T_{0}$ : Equations (13)(16) can be valid even for large deviations from the initial values, i.e., for large distances, assuming that perturbation functions $g_{i}$ in Eqs. (9)-(12) depend on the current values of soliton parameters rather than on the initial values. Therefore, to study soliton statistics for large distances and to determine the probabilities of large fluctuations given by the tails of PDEs, one needs to recognize the system of Eqs. (13)-(16) as a system with multiplicative noise because projection functions $g_{i}$ in Eq. (17) 
depend on the stochastic variables $A, \phi, \Omega, T$ by means of Eqs. (9)-(12). This fact was probably first emphasized in Ref. 2. The majority of the results obtained so far were obtained under the implicit assumption that functions $g_{i}$ do not depend on the variables or rather depend on the initial values $A_{0}, \phi_{0}, \Omega_{0}$, and $T_{0}$. Under such an assumption $S_{i}$ become independent AWGNs, and one can readily calculate their variances. With $\mathrm{AWGN} S_{i}$ the statistics of the solutions of Eqs. (13)-(16) becomes Gaussian, and the variances of those Gaussian distributions give well-known results. ${ }^{6}$

Considering the problem of random perturbations to the NLSE, Eq. (18) at first glance seems to be ambiguous: It admits of two different interpretations, namely, those of Ito and of Stratonovich. ${ }^{23}$ The two interpretations are equally acceptable from the mathematical point of view. However, they produce different results for the soliton statistics, and the corresponding FPEs differ for the two approaches (by an advection term). In the Ito approach the functions $g_{i}$ on the right-hand side of Eq. (18) are assumed to be statistically independent of the noise. That significantly simplifies the reasoning because at each point $z$ one can treat each noise part in Eq. (18) as real uncorrelated Gaussian noise with zero mean and variance $n_{i}$, depending on the current values of the soliton parameters:

$$
n_{i}(\mathbf{q})=\frac{D}{2} \int_{-\infty}^{\infty}\left|g_{i}(t, \mathbf{q})\right|^{2} \mathrm{~d} t
$$

In the Stratonovich approach the noise term on the righthand side of Eq. (18) is symmetrically regularized in distance $z,^{15,23}$ and it no longer decouples from projection function $g_{i}$.

In the research reported in Refs. 17 and 18 the Ito approach was used, whereas in Ref. 19 the Stratonovich interpretation was applied to derive the FPE for soliton parameters.

The two approaches gave different results for the PDF for the amplitude of the soliton. Here we clarify this issue. It is important to note that the initial model for our soliton system is not the Langevin system with multiplicative noise but rather the modified NLSE with additive noise [Eq. (1)]. For systems with additive noise the ItoStratonovich dilemma simply does not exist: both approaches should yield the same results. ${ }^{15,23}$ However, the soliton perturbation theory brings forth a system with multiplicative noise that, at first glance, produces different results that depend on the approach chosen. An explanation of this issue is rather simple: The perturbation theory for the soliton parameters is different for the two approaches. One has to bear in mind that the rules of the Ito calculus are different from those of normal calculus (which can still be applied in the Stratonovich approach). In particular, the ordinary chain rule for differentiation no longer applies to Ito calculus. For an arbitrary function $f(\mathbf{q}, z)$, where vector $\mathbf{q}$ is a solution of Eq. (18), the Ito chain rule reads as

$$
\begin{aligned}
\frac{\mathrm{d} f}{\mathrm{~d} z}=\frac{\partial f}{\partial z} & +\sum_{i} \frac{\partial f}{\partial q_{i}} \frac{\mathrm{d} q_{i}}{\mathrm{~d} z} \\
& +\frac{D}{4} \operatorname{Re} \sum_{i, k}\left[\int_{-\infty}^{\infty} \mathrm{d} t g_{i}^{*}(t, \mathbf{q}) g_{k}(t, \mathbf{q})\right] \frac{\partial^{2} f}{\partial q_{i} \partial q_{k}} .
\end{aligned}
$$

We can prove this formula by introducing the real and imaginary parts of complex noise $\eta$ and discrete time $t_{j}$. Equation (18) then becomes discrete in time and real valued. For such a system the multidimensional Ito chain rule can be found in Refs. 23 and 24. The last term in Eq. (20) reveals the difference between Ito calculus and ordinary calculus. However, the adiabatic soliton perturbation theory used to drive Eqs. (5)-(8) was based on the assumption of ordinary calculus (see, for example, Ref. 6). In particular, the solution of perturbed NLSE (1) is sought in the form

$$
u(z, t)=u_{0}[A(z), \phi(z), \Omega(z), T(z) ; t]+u_{\mathrm{rad}},
$$

where $u_{0}$ is a single-soliton ansatz with adiabatically slowly varying parameters and $u_{\text {rad }}$ is a radiative part (continuum). One has to insert Eq. (21) into Eq. (1) and perform linearization [see formula (5.53) of Ref. 6]. However, during such a linearization a conventional chain rule is used, which is inconsistent with the Ito rule [Eq. (20)]. Therefore the ordinary perturbation theory is inapplicable to the Ito stochastic perturbations unless some changes are made. Stratonovich calculus, however, uses the same symbolic rules as does ordinary calculus, and therefore the perturbation analysis of Ref. 6 and Eqs. (5)(8) is still valid, provided that the Stratonovich convention is used throughout.

So we have shown that Eqs. (5)-(8) must be used with the Stratonovich convention and not that of Ito. However, one can still employ Ito calculus for studying the statistics of soliton parameters, provided that extra advection terms are inserted into the system of Eqs. (5)-(8). Indeed, the Stratonovich system [Eq. (18)] is equivalent to an Ito system in which the following substitution has been made:

$$
f_{i}(\mathbf{q}) \rightarrow f_{i}(\mathbf{q})+\frac{D}{4} \operatorname{Re} \int \mathrm{d} t \sum_{j} g_{j} *(t, \mathbf{q}) \frac{\partial g_{i}(t, \mathbf{q})}{\partial q_{j}}
$$

Using perturbation functions $g_{i}$ given by Eqs. (9)-(12), one can show that the only equation that changes in the Ito representation is Eq. (5). It reads as

$$
\frac{\mathrm{d} A}{\mathrm{~d} z}=D+\operatorname{Re} \int \mathrm{d} t g_{A} *(t) \eta(z, t) .
$$

The remaining equations are the same as Eqs. (6)-(8).

This extra advection term partially explains the discrepancies between soliton amplitude statistics in Refs. 17 and 19 and the correct results given in Ref. 19.

\section{FOKKER-PLANCK EQUATION}

For the most general system, such as Eqs. (13)-(16), it is possible to write a FPE for the joint PDF, 
$P(A, \phi, \Omega, T \mid z)$. For our system of Eqs. (13)-(16) the FPE has the following form (see Appendix A):

$$
\begin{aligned}
\frac{\partial P}{\partial z}= & -\frac{1}{2}\left(A^{2}-\Omega^{2}\right) \frac{\partial P}{\partial \phi}+\frac{\partial}{\partial T}\{\Omega P\}-\frac{\partial}{\partial A}\{D P+\alpha A P \\
& \left.-\eta\left(\Omega^{2}+\frac{A^{2}}{3}\right) A P\right\}+\frac{\partial}{\partial \Omega}\left\{\frac{2}{3} \eta A^{2} \Omega P\right\} \\
& +D\left\{\frac{1}{6} A T^{2}+\frac{1}{12 A}\left(2+\frac{\pi^{2}}{6}\right)\right\} \frac{\partial^{2} P}{\partial \phi^{2}} \\
& +\frac{D}{3} A T \frac{\partial^{2} P}{\partial \phi \partial \Omega}+\frac{D}{2} \frac{\partial^{2}}{\partial A^{2}}[A P] \\
& +\frac{D}{6} A \frac{\partial^{2} P}{\partial \Omega^{2}}+\frac{D \pi^{2}}{24 A^{3}} \frac{\partial^{2} P}{\partial T^{2}} .
\end{aligned}
$$

At the origin, $z=0$, soliton parameters have deterministic values, so $P(A, \phi, \Omega, \phi \mid 0)=\delta\left(A-A_{0}\right) \delta(\phi$ $\left.-\phi_{0}\right) \delta\left(T-T_{0}\right) \delta\left(\Omega-\Omega_{0}\right)$. It is desirable that we choose initial parameters close to the stationary solution of Eqs. (13)-(16) without noise, because doing so will ensure the stability of soliton propagation. A simple analysis of Eqs. (13)-(16) shows ${ }^{6}$ that stationary frequency $\Omega_{s}$ is zero and that stationary soliton position $T_{s}$ is an arbitrary constant, whereas for the stationary amplitude we have the relation $\alpha=\eta A_{s}{ }^{2} / 3$. A stationary solution for phase $\phi$ does not exist; however, if we renormalize the phase to include stationary self-phase modulation, $\widetilde{\phi}$ $=\left(\phi-A_{s}{ }^{2} z / 2\right) \bmod 2 \pi$, the quantity $\widetilde{\phi}_{s}$ is an arbitrary constant from 0 to $2 \pi$. Therefore we choose $\Omega_{0}=T_{0}$ $=0$. And the requirement that $A_{0}$ be a stationary solution will fix the value of excess gain $\alpha$, which so far has been arbitrary:

$$
\alpha=\eta A_{0}^{2} / 3 \text {. }
$$

We also choose $\phi_{0}=\widetilde{\phi}_{0}=0$. The boundary conditions in $\Omega$ and $T$ are as follows: $P$ should decrease rapidly for $|\Omega| \rightarrow \infty,|T| \rightarrow \infty$. In $\phi$ the boundary conditions are those of periodicity: $\quad P(A, \phi+2 \pi, \Omega, T \mid z)$ $=P(A, \phi, \Omega, T \mid z)$, with $0 \leqslant \phi<2 \pi$. As for amplitude $A$, we assume that the $A$ component of probability current vector $\mathbf{j}$ is zero everywhere at plane $A=0$ :

$$
\begin{aligned}
\left.j_{A}\right|_{A=0}= & \left\{D P+\alpha A P-\eta\left(\Omega^{2}+\frac{A^{2}}{3}\right) A P\right. \\
& \left.-\frac{D}{2} \frac{\partial}{\partial A}[A P]\right\}\left.\right|_{A=0}=0 .
\end{aligned}
$$

Such a boundary condition ensures that we neglect soliton creation and annihilation, which are beyond the scope of applicability of the adiabatic soliton perturbation theory anyway. Because at $A=0$ function $P$ may have an integrable singularity only, it follows that always

$$
P(0, \phi, \Omega, T \mid z)=0 \text {. }
$$

Equation (24) is quite complicated and not easy to solve. Therefore we dedicate Section 5 below to specific cases for which we can find exact solutions for (marginal) PDFs, whereas in the rest of the paper we consider some approximate methods of solving Eq. (24).

\section{EXACT SOLUTION FOR AMPLITUDE- FREQUENCY JITTER: SYSTEMS WITHOUT FILTERING}

Equation (24) cannot be solved analytically in general. However, often we are not interested in the full PDF $P(A, \phi, \Omega, T \mid z)$ but rather in some marginal distribution functions. In some cases we can obtain an autonomous equation for the marginal PDF by integrating out the redundant degrees of freedom in Eq. (24). Here we derive and solve such an autonomous equation for a marginal PDF for amplitude and frequency only, $P(A, \Omega \mid z)$, in the system without filtering (i.e., $\alpha=\eta=0$ ). The corresponding FPE describes soliton statistics for a pure NLSE with AWGN:

$$
\begin{aligned}
\frac{\partial P}{\partial z}= & -\frac{1}{2}\left(A^{2}-\Omega^{2}\right) \frac{\partial P}{\partial \phi}+\frac{\partial}{\partial T}\{\Omega P\}-\frac{\partial}{\partial A}\{D P\} \\
& +D\left\{\frac{1}{6} A T^{2}+\frac{1}{12 A}\left(2+\frac{\pi^{2}}{6}\right)\right\} \frac{\partial^{2} P}{\partial \phi^{2}} \\
& +\frac{D}{3} A T \frac{\partial^{2} P}{\partial \phi \partial \Omega}+\frac{D}{2} \frac{\partial^{2}}{\partial A^{2}}[A P]+\frac{D}{6} A \frac{\partial^{2} P}{\partial \Omega^{2}} \\
& +\frac{D \pi^{2}}{24 A^{3}} \frac{\partial^{2} P}{\partial T^{2}} .
\end{aligned}
$$

Now we can integrate this equation over $\phi$ and $T$, using integration by parts and boundary conditions in $\phi$ and $T$. We arrive at a simpler equation for the marginal PDF, $P(A, \Omega \mid z)$ :

$$
\frac{\partial P}{\partial z}=\frac{D}{2} A \frac{\partial^{2} P}{\partial A^{2}}+\frac{D}{6} A \frac{\partial^{2} P}{\partial \Omega^{2}} .
$$

The initial condition reads as

$$
P(\Omega, A \mid 0)=\delta(\Omega) \delta\left(A-A_{0}\right) .
$$

The boundary conditions in $\Omega$ are the usual ones, which provide a rapid decrease of $P$ as $|\Omega|$ goes to infinity. For the amplitude we write, following Eq. (26),

$$
\left.P\right|_{A=0}=0,\left.\quad P\right|_{A \rightarrow \infty} \rightarrow 0 .
$$

As $A$ goes to infinity, $P$ should decrease rapidly to provide normalization. To solve Eq. (28) we apply a Fourier transform with respect to $\Omega$ and a Laplace transform with respect to $A$ :

$$
P_{k}(\lambda \mid z)=\int_{-\infty}^{\infty} \mathrm{d} \Omega \int_{0}^{\infty} \mathrm{d} A P(A, \Omega \mid z) \exp (-\lambda A+i k \Omega) .
$$

Then, after the transformation, the equation takes the form

$$
\frac{\partial P_{k}}{\partial z}=D\left(\frac{k^{2}}{6}-\frac{\lambda^{2}}{2}\right) \frac{\partial P_{k}}{\partial \lambda}-D \lambda P_{k} .
$$


The initial condition is $P_{k}(\lambda \mid 0)=\exp \left(-\lambda A_{0}\right)$. Equation (31) can be solved by the method of characteristics. Omitting the details, we present the result in the form

$$
P(\Omega, A \mid z)=\frac{1}{2 \pi} \int_{-\infty}^{\infty} P_{k}\left(A \mid z^{\prime}\right) \exp (-i k \Omega) \mathrm{d} k,
$$

where

$$
\begin{aligned}
P_{k}\left(A \mid z^{\prime}\right)= & \left(\frac{A}{A_{0}}\right)^{1 / 2} \frac{\alpha_{k}}{\sinh \left(\alpha_{k} z^{\prime}\right)} \exp \left[-\alpha_{k}(A\right. \\
& \left.\left.+A_{0}\right) \operatorname{coth}\left(\alpha_{k} z^{\prime}\right)\right] I_{1}\left[\frac{2 \alpha_{k} \sqrt{A A_{0}}}{\sinh \left(\alpha_{k} z^{\prime}\right)}\right] .
\end{aligned}
$$

$I_{1}$ is the modified Bessel function, $z^{\prime}=(D / 2) z$, and $\alpha_{k}$ $=k / \sqrt{3}$.

Integrating Eq. (32) over frequency we obtain the PDF for soliton amplitude jitter $P(A)=\int P(\Omega, A \mid z) \mathrm{d} \Omega$ :

$$
\begin{aligned}
P\left(A \mid z^{\prime}\right) & =P_{0}\left(A \mid z^{\prime}\right) \\
& =\frac{1}{z^{\prime}}\left(\frac{A}{A_{0}}\right)^{1 / 2} \exp \left(-\frac{A+A_{0}}{z^{\prime}}\right) I_{1}\left(\frac{2 \sqrt{A A_{0}}}{z^{\prime}}\right) .
\end{aligned}
$$

Equation (34) represents an explicit form of the nonGaussian PDF, $P(A)$. Using an asymptotic expansion for the Bessel function, one can verify that $P(A)$ has an exponential tail, as was predicted in Ref. 13:

$$
P(A) \sim\left(\frac{A}{A_{0}}\right)^{1 / 2} \exp \left[-\frac{\left(\sqrt{A}-\sqrt{A_{0}}\right)^{2}}{z^{\prime}}\right], \quad A \rightarrow \infty .
$$

Now we can use the exact $\operatorname{PDF}, P\left(A \mid z^{\prime}\right)$, to calculate higher-order moments:

$$
\left\langle A^{n}\right\rangle=z^{\prime n} n ! L_{n}^{1}\left(-A_{0} / z^{\prime}\right),
$$

where $L_{n}{ }^{1}$ are the generalized Laguerre functions. Note the expression for the first-order moment, $\langle A\rangle=A_{0}$ $+D z$, which implies a systematic increase of the soliton amplitude as a result of the noise. This fact had been overlooked in the literature until recently and was pointed out in Ref. 13. We can also calculate the moments for the frequency $\Omega$ by using Eqs. (32) and (33) (the only nonvanishing moments are even ones):

$$
\begin{aligned}
\left\langle\Omega^{2 n}\right\rangle & =\left.(-1)^{n} \int_{0}^{\infty} \mathrm{d} A \frac{\partial^{2 n} P_{k}\left(A \mid z^{\prime}\right)}{\partial k^{2 n}}\right|_{k=0} \\
& =\left.(-1)^{n} \frac{\partial^{2 n} G\left(k \mid z^{\prime}\right)}{\partial k^{2 n}}\right|_{k=0},
\end{aligned}
$$

where generating function $G$ has the form

$$
G\left(k \mid z^{\prime}\right)=\operatorname{sech}^{2}\left(\alpha_{k} z^{\prime}\right) \exp \left[-\alpha_{k} \tanh \left(\alpha_{k} z^{\prime}\right) A_{0}\right] .
$$

In particular, for the variance we have

$$
\left\langle\Omega^{2}\right\rangle=\frac{D A_{0} z}{3}+\frac{D^{2} z^{2}}{6} .
$$

If we compare this result with that obtained with the Gaussian approximation [see formula (5.113) of Ref. 6] we observe a correction of higher order in $D z$, which stems from the non-Gaussian statistics.

Equations (32) and (34) represent accurate results for the marginal PDFs obtained directly from the FPE. We use these results in the subsequent sections for comparison with results obtained by the approximate methods.

\section{WEAK-NOISE LIMIT: WKB APPROXIMATION}

FPE (24) is a special case of a generic FPE [Eq. (A3) of Appendix A], with advection and diffusion coefficients given by Eqs. (A4) and (A5). Here our goal is to build a perturbation theory for the corresponding FPE [Eq. (A3)] for small values of noise $D$. We use the WKB method and the procedure described in Ref. 25.

We search for a solution in the form

$$
P=\exp \left(-\frac{S}{D}\right)
$$

where the function $S$ is an effective action, and we expand it in powers of $D$ :

$$
S=S_{0}+D S_{1}+D^{2} S_{2}+\ldots
$$

and then substitute it into Eq. (A3). In leading order in $D$ we obtain a Hamiltonian-Jacobi equation for the function $W=S_{0}$ :

$$
\frac{\partial W}{\partial z}+\sum_{i} F^{i} \frac{\partial W}{\partial q_{i}}+\sum_{i j} Q^{i j} \frac{\partial W}{\partial q_{i}} \frac{\partial W}{\partial q_{j}}=0
$$

where

$$
\begin{gathered}
F^{i}=\lim _{D \rightarrow 0} D_{i}, \\
Q^{i j}=\lim _{D \rightarrow 0} \frac{D_{i j}}{D} .
\end{gathered}
$$

The solution of Eq. (39) is obtained in terms of the Lagrange function

$$
W=\int L \mathrm{~d} z=\int \mathbf{p} \mathrm{d} \mathbf{q}-H \mathrm{~d} z,
$$

where the Lagrangian is given by

$$
L\left(\mathbf{q}, \mathbf{q}^{\prime}\right)=1 / 4 Q_{i j}(\mathbf{q})\left(q^{\prime i}-F^{i}\right)\left(q^{\prime j}-F^{j}\right),
$$

where $Q_{i j}$ is the inverse of matrix $Q^{i j}$ and primes denote differentiation with respect to $z$. It has the following form: 


$$
=\left[\begin{array}{cccc}
2 / A & 0 & 0 & 0 \\
0 & \frac{72 A}{12+\pi^{2}} & -\frac{72 A T}{12+\pi^{2}} & 0 \\
0 & -\frac{72 A}{12+\pi^{2}} & \frac{6}{A} \frac{12+\pi^{2}+12 A^{2} T^{2}}{12+\pi^{2}} & 0 \\
0 & 0 & 0 & \frac{24 A^{3}}{\pi^{2}}
\end{array}\right] .
$$

Momenta $p_{i}$ are introduced as canonically conjugated variables of $q_{i}$ :

$$
p_{i}=\frac{\partial L}{\partial q^{\prime i}}=\frac{\partial W}{\partial q^{i}},
$$

and the effective Hamiltonian is

$$
H(\mathbf{q}, \mathbf{p})=Q^{i j}(\mathbf{q}) p_{i} p_{j}+F^{i}(\mathbf{q}) p_{i} .
$$

(Einstein summation rules are implied here and throughout). The solution of Hamilton-Jacobi equation (39) is the action taken along the Hamiltonian trajectories, which are given as solutions of Hamilton's equations:

$$
\begin{aligned}
& \frac{\mathrm{d} q^{i}}{\mathrm{~d} z}=\frac{\partial H}{\partial p_{i}}=F^{i}+2 Q^{i j}(\mathbf{q}) p_{j} \\
& \frac{\mathrm{d} p_{i}}{\mathrm{~d} z}=-\frac{\partial H}{\partial q^{i}}=-\frac{\partial F^{j}(\mathbf{q})}{\partial q^{i}} p_{j}-\frac{\partial Q^{j k}(\mathbf{q})}{\partial q^{i}} p_{j} p_{k} .
\end{aligned}
$$

We are interested in the special solution of the FPE that has deterministic values $\mathbf{q}_{0}=\left(A_{0}, \phi_{0}, \Omega_{0}, T_{0}\right)$ at the origin $z=0$. Such a solution $P\left(\mathbf{q} \mid \mathbf{q}_{0} ; Z\right)$ is called a propagator and in the weak-noise limit is given by ${ }^{25}$

$$
\begin{aligned}
P\left(\mathbf{q} \mid \mathbf{q}_{0} ; Z\right)= & \frac{1}{(2 \pi D)^{2}}\left|\operatorname{det}\left(-\frac{\partial^{2} W}{\partial \mathbf{q} \partial \mathbf{q}_{0}}\right)\right|^{1 / 2} \\
& \times \exp \left(\int_{0}^{Z} \mathbf{G} \mathbf{p d} z\right) \\
& \times \exp \left(-\frac{1}{2} \int_{0}^{Z} \operatorname{Tr} \frac{\partial^{2} H}{\partial \mathbf{q} \partial \mathbf{p}} \mathrm{d} z\right) \\
& \times \exp \left[-\frac{1}{D} W\left(\mathbf{q}, \mathbf{q}_{0} ; Z\right)\right],
\end{aligned}
$$

where

$$
\mathbf{G}=\lim _{D \rightarrow \infty} \frac{1}{D} D_{i}
$$

and $W\left(\mathbf{q}, \mathbf{q}_{0} ; Z\right)$ is the action of the Hamiltonian trajectory from $\mathbf{q}_{0}$ to $\mathbf{q}$ during the interval $Z$. The Hamilton equations for the system of Eqs. (13)-(16) read as

$$
\frac{\mathrm{d} A}{\mathrm{~d} z}=\alpha A-\eta\left(\Omega^{2}+A^{2} / 3\right) A+A p_{A},
$$

$$
\begin{aligned}
\frac{\mathrm{d} \phi}{\mathrm{d} z}= & (1 / 2)\left(A^{2}-\Omega^{2}\right)+\left[\frac{1}{3} A T^{2}+\frac{1}{6 A}\right. \\
& \left.\times\left(2+\frac{\pi^{2}}{6}\right)\right] p_{\phi}+(A T) / 3 p_{\Omega}, \\
\frac{\mathrm{d} \Omega}{\mathrm{d} z}= & -(2 / 3) \eta A^{2} \Omega+(A T / 3) p_{\phi}+(A / 3) p_{\Omega}, \\
\frac{\mathrm{d} T}{\mathrm{~d} z}= & -\Omega+\frac{\pi^{2}}{12 A^{3}} p_{T},
\end{aligned}
$$

$\frac{\mathrm{d} p_{A}}{\mathrm{~d} z}=\left[-\alpha+\eta\left(A^{2}+\Omega^{2}\right)\right] p_{A}-A p_{\phi}$

$$
+(4 / 3) \eta A \Omega_{p \Omega}-(1 / 2) p_{A}^{2}-\left[(1 / 6) T^{2}\right.
$$$$
\left.-\frac{1}{12 A^{2}}\left(2+\frac{\pi^{2}}{6}\right)\right] p_{\phi}{ }^{2}-(1 / 6) p_{\Omega}{ }^{2}
$$$$
+\frac{\pi^{2}}{8 A^{4}} p_{T}^{2}
$$

$$
\frac{\mathrm{d} p_{\phi}}{\mathrm{d} z}=0,
$$

$$
\frac{\mathrm{d} p_{\Omega}}{\mathrm{d} z}=2 \eta \Omega A p_{A}+\Omega p_{\phi}+(2 / 3) \eta A^{2} p_{\Omega}+p_{T},
$$

$$
\frac{\mathrm{d} p_{T}}{\mathrm{~d} z}=-(1 / 3) A T{p_{\phi}}^{2} .
$$

The trajectories in Eq. (45) are the solutions of Eqs. (46) (53) subject to the following boundary conditions:

$$
\begin{array}{ll}
A(0)=A_{0}, & A(Z)=A, \\
\phi(0)=\phi_{0}, & \phi(Z)=\phi, \\
\Omega(0)=\Omega_{0}, & \Omega(Z)=\Omega, \\
T(0)=T_{0}, & T(Z)=T .
\end{array}
$$

Without loss of generality we may assume that $\phi_{0}$ $=T_{0}=0$. The Hamiltonian system above possesses two types of stationary state: those with $\mathbf{p}=0$ and those with $\mathbf{p} \neq 0$. The former are the stationary solutions of Eqs. (13)-(16) without noise that were considered in Section 4. Henceforth we are interested only in these stationary solutions.

\section{A. Classic Results: Gordon-Haus Formula}

In this section we calculate the PDF when propagation distance $z$ is not large and also when the arguments of the PDF are close to the initial values; i.e., we describe the bulk of the PDF for short distances. Under such conditions Eq. (44) can be linearized about the stationary solution $\mathbf{q}=\mathbf{q}_{s}, \mathbf{p}=0$ : 


$$
\begin{aligned}
& \frac{\mathrm{d} \delta \mathbf{q}}{\mathrm{d} z}=\hat{L} \delta \mathbf{q}+2 \hat{\mathbf{Q}} \mathbf{p}, \\
& \frac{\mathrm{d} \mathbf{p}}{\mathrm{d} z}=-\hat{L}^{T} \mathbf{p},
\end{aligned}
$$

where $\quad \delta \mathbf{q}=\mathbf{q}-\mathbf{q}_{s}, \quad \hat{\mathbf{Q}}=\left[Q^{i j}\left(\mathbf{q}_{s}\right)\right], \quad$ and $L_{j}^{i}=\partial F^{i}\left(\mathbf{q}_{s}\right) / \partial q^{j}$. The PDF of the linearized problem is Gaussian. It was calculated in Ref. 25:

$$
\begin{aligned}
& P\left(\delta \mathbf{q}, \delta \mathbf{q}_{0} ; z\right) \\
& \quad=\frac{1}{(4 \pi D)^{2}} \frac{1}{[\operatorname{det} \hat{R}(z)]^{1 / 2}} \exp \left(-\frac{1}{4 D} \delta \mathbf{q}^{T} \hat{R}^{-1} \delta \mathbf{q}\right),
\end{aligned}
$$

where matrix $\hat{R}(z)$ is defined as

$$
\hat{R}(z)=\int_{0}^{z} \mathrm{~d} z^{\prime} \exp \left(\hat{L} z^{\prime}\right) \hat{Q} \exp \left(\hat{L}^{T} z^{\prime}\right) .
$$

The variances of the soliton parameters are given by the diagonal components of matrix $\hat{R}$. For Eqs. (46)-(53) we obtain

$$
\begin{aligned}
\left\langle\delta A(z)^{2}\right\rangle= & 2 D R_{A A}=\frac{D A_{0}}{2 \beta}[1-\exp (-2 \beta z)], \quad(58) \\
\left\langle\delta \phi(z)^{2}\right\rangle= & \frac{A_{0}^{3} D}{2 \beta^{3}}[2 \beta z+4 \exp (-\beta z) \\
& -\exp (-2 \beta z)-3]+\frac{\mathrm{D} z}{3 A_{0}}\left(1+\frac{\pi^{2}}{12}\right), \\
\left\langle\delta \Omega(z)^{2}\right\rangle= & \frac{A_{0} D}{6 \beta}[1-\exp (-2 \beta z)], \\
\left\langle\delta T(z)^{2}\right\rangle= & \frac{A_{0} D}{6 \beta^{3}}[4 \exp (-\beta z)-\exp (-2 \beta z) \\
& -3+2 \beta z]+\frac{D \pi^{2} z}{12 A_{0}^{3}},
\end{aligned}
$$

where $\beta=(2 / 3) \eta A_{0}{ }^{2}$. These results coincide with those given in Ref. 6 . In particular, the formula for timing jitter $\left\langle\delta T^{2}\right\rangle$ coincides with the classic Gordon-Haus result ${ }^{1}$ in the limit of zero filtering, $\beta \rightarrow 0$. So we have obtained classic results for Gaussian statistics of the soliton parameters for small propagation distances $z$ and for the core of the PDF.

\section{B. Asymptotic Solution for the Tails of the Probability- Density Function}

The Hamiltonian system of Eqs. (46)-(53) in general cannot be solved analytically. Therefore we make a series of simplifying assumptions here that will allow us to obtain some results for the PDF in closed form. First, note that phase $\phi$ does not enter into the coefficients of FPE (A3) and is a cyclic coordinate in Hamiltonian (43). This means that if one is interested not in the statistics of phase $\phi$ but only in the marginal PDF for the rest of the soliton parameters $P(A, \Omega, T ; Z)$ $=\int P(A, \Omega, T, \phi \mid Z) \mathrm{d} \phi$ we can obtain a closed FokkerPlanck equation for such a PDF by simply integrating Eq. (A3) by parts and using periodic boundary conditions. For the effective Hamiltonian description that we adopt, this means that we obtained a reduced Hamiltonian system that does not contain the equation for the phase and $p_{\phi}$ is set to zero. However, the system obtained is still too complicated, and one needs to simplify it even further to obtain analytical results. We assume large values of the amplitude, $A \gg 1$. In this limit, neglecting the terms $\sim A^{-n}, n>0$, we obtain the following system:

$$
\begin{aligned}
& \frac{\mathrm{d} A}{\mathrm{~d} z}=\alpha A-\eta\left(\Omega^{2}+A^{2} / 3\right) A+A p_{A} \\
& \frac{\mathrm{d} \Omega}{\mathrm{d} z}=-(2 / 3) \eta A^{2} \Omega+(A / 3) p_{\Omega} \\
& \frac{\mathrm{d} T}{\mathrm{~d} z}=-\Omega \\
& \frac{\mathrm{d} p_{A}}{\mathrm{~d} z}=\left[-\alpha+\eta\left(A^{2}+\Omega^{2}\right)\right] p_{A}-(4 / 3) \eta A \Omega p_{\Omega} \\
& \frac{\mathrm{d} p_{\Omega}}{\mathrm{d} z}=2 \eta \Omega A p_{A}+(2 / 3) \eta A^{2} p_{\Omega}+p_{T} \\
& \frac{\mathrm{d} p_{T}}{\mathrm{~d} z}=0
\end{aligned}
$$

Here we consider the simplest case when the filtering is absent: $\alpha=\eta=0$. In this case equations for the momenta become decoupled from those for the soliton parameters. Equations for $p_{T}$ and $p_{\Omega}$ yield

$$
\begin{aligned}
& p_{T}(z)=p_{T_{0}}, \\
& p_{\Omega}(z)=p_{T_{0}} z+p_{\Omega_{0}} .
\end{aligned}
$$

The equation for $p_{A}$ is a Riccati equation and does not have a compact-form solution. Therefore we assume here that $p_{\Omega} \ll p_{A}$ and later provide the necessary applicability conditions. Then the solution for the momentum $p_{A}$ is given by

$$
p_{A}(z)=\frac{2 p_{A_{0}}}{2+p_{A_{0}} z} .
$$

The solution of the amplitude equation that satisfies boundary conditions (54) is given by

$$
A(z)=\left[\sqrt{A_{0}}+\left(\sqrt{A}-\sqrt{A_{0}}\right) \frac{z}{Z}\right]^{2},
$$

while momentum $p_{A}$ is

$$
p_{A}(z)=2 \frac{\sqrt{A}-\sqrt{A_{0}}}{\sqrt{A_{0}} Z+\left(\sqrt{A}-\sqrt{A_{0}}\right) z} .
$$


The remaining equations for $T(z)$ and $\Omega(z)$ subject to boundary conditions (54) can be solved. The expressions for the Hamiltonian trajectories are quite bulky and are not given here. In particular, one can find constants $p_{T_{0}}$ and $p_{\Omega_{0}}$ that in the leading order in $A^{-1}$ are

$$
\begin{aligned}
& p_{\Omega_{0}}=\frac{180 T}{A Z^{2}}-\frac{36 \Omega}{A Z}, \\
& p_{T_{0}}=\frac{240 T}{A Z^{3}}+\frac{60 \Omega}{A Z^{2}} .
\end{aligned}
$$

The applicability condition for the above analysis is

$$
\left|\frac{p_{A}}{p_{\Omega}}\right| \sim \max \left[\frac{A Z^{2}}{240 T}, \frac{A Z}{60 \Omega}\right] \gg 1 ;
$$

i.e., the found solution of the Hamiltonian system is valid for values of $\Omega$ and $T$ that are not large.

Next we need to calculate the action defined by Eq. (40). It consists of two parts: the conserved Hamiltonian (pseudoenergy) and the reduced action. We calculate the contribution of each part separately. Conserved Hamiltonian $H$ [Eq. (43)] for the reduced system without phase and filtering reads as

$$
H=E=-\Omega p_{T}+\frac{1}{2} A p_{A}{ }^{2}+\frac{1}{6} A p_{\Omega}{ }^{2}+\frac{\pi^{2}}{24 A^{3}} p_{T}^{2} .
$$

Because the energy is an integral of motion, it can be evaluated at the origin $z=0$. Because of boundary conditions (54), the first term is zero. The third term can be neglected under condition (73), and the fourth term can be omitted, provided that $z \gtrsim 1$. The main contribution comes from the second term, and it can be written as

$$
W_{E}=-\frac{\left(\sqrt{A}-\sqrt{A_{0}}\right)^{2}}{Z / 2} .
$$

The calculation of reduced action $W^{\prime}$ is more involved but can be done, producing in the leading order in $A^{-1}$

$$
W^{\prime}=\frac{\left(\sqrt{A}-\sqrt{A_{0}}\right)^{2}}{Z / 4}+\frac{240 T^{2}}{A Z^{3}}+\frac{60 T \Omega}{A Z^{2}} .
$$

From Eq. (45) we can conjecture that, up to a prefactor, the asymptote sought for the PDF is

$$
\begin{aligned}
\ln P(A, T, \Omega \mid Z) \approx & -W / D \approx-\frac{\left(\sqrt{A}-\sqrt{A_{0}}\right)^{2}}{D Z / 2}-\frac{240 T^{2}}{D A Z^{3}} \\
& +\frac{60 T \Omega}{D A Z^{2}}, \quad A \gg A_{0},
\end{aligned}
$$

where condition (73) is assumed as well as $z \gtrsim 1$. This result is in excellent agreement with the asymptote for the marginal PDF $P(\mathrm{~A})$ given by relation (35). It also shows that for large values of $A$ the tails of the PDF are Gaussian in $T$. However, this does not mean that the marginal statistics of the soliton position are Gaussian, because these statistics are determined by the behavior of the PDF for arbitrary values of $A$ and not only for large values.

\section{DISCUSSION}

The equations of adiabatic perturbation theory for soliton parameters in the presence of ASE are Langevin equations with multiplicative noise. They give rise to the non-Gaussian statistics of soliton parameters. These statistics can be adequately described by the Stratonovich Fokker-Planck equation (or the Ito Fokker-Planck equation, provided that the advection vector is redefined). In this paper we have derived a generic FPE for all four soliton parameters in the presence of in-line filtering. Although it is not possible to solve this equation in general, except in a few special cases that we have considered, it is still possible to obtain asymptotic results for small noise intensity $D$ by using the WKB method. In this approach one has to solve a boundary problem for a system of second-order ordinary differential equations (46)-(54) instead of partial differential equation (24). We used this approach to derive the tails of the joint $\operatorname{PDF} P(A, \Omega, T \mid z)$ in the absence of filtering. The WKB approach can also be beneficial when one is considering a numerical solution of the problem. The classic formulas for the GordonHaus effect and Gaussian statistics are recovered from the FPE in the limit of small propagation distance $z$. A joint PDF for frequency and amplitude jitter was calculated from the FPE and has proved to be non-Gaussian. Note that any other control devices, e.g., Butterworth filters and amplitude and phase modulators, can be incorporated into the problem. The only restriction is that they have to be treated perturbatively as required by adiabatic soliton perturbation theory. Adding such devices will change the advection terms of the FPE, which will affect the steady state of the system.

\section{APPENDIX A: DERIVATION OF THE FOKKER-PLANCK EQUATION BY USE OF THE FURUTSU-NOVIKOV FORMULA}

The system of Eqs. (13)-(16) is a special case of a general system with complex white Gaussian multiplicative noise [Eq. (18)], where $\mathbf{q}$ is an $N$-component real field, $f_{i}$ is a deterministic advection term, $g_{i}$ are the projection functions that themselves depend on the random field $\mathbf{q}$, and, finally, $\chi$ is complex white Gaussian noise with statistical properties given by Eqs. (2) and (3). Here we assume the Stratonovich interpretation of the Langevin system. We are interested in the equation that governs the evolution of the PDF:

$$
P(\mathbf{q} \mid z)=\left\langle\prod_{i=1}^{N} \delta\left[q_{i}-q_{i}^{s}(z)\right]\right\rangle,
$$

where $\mathbf{q}_{s}$ is the solution of the Langevin system [Eq. (18)] Differentiating formula (A1) with respect to $z$, we obtain

$$
\frac{\partial P}{\partial z}=-\sum_{j}\left\langle\frac{\partial}{\partial q_{j}} \prod_{i} \delta\left[q_{i}-q_{i}{ }^{s}(z)\right] \frac{\mathrm{d} q_{j}{ }^{s}}{\mathrm{~d} z}\right\rangle,
$$


where we can now substitute the derivative $\mathrm{d} q_{j}{ }^{s} / \mathrm{d} z$ directly from Eq. (18):

$$
\begin{aligned}
\frac{\partial P}{\partial z}= & -\sum_{j}\left\langle\frac { \partial } { \partial q _ { j } } \prod _ { i } \delta [ q _ { i } - q _ { i } ^ { s } ( z ) ] \left[ f_{j}\left(\mathbf{q}^{s}\right)\right.\right. \\
& \left.\left.+\operatorname{Re} \int_{-\infty}^{\infty} \mathrm{d} t g_{j}^{*}\left(\mathbf{q}_{s}, t\right) \chi(z, t)\right]\right\rangle \\
= & -\sum_{j} \frac{\partial}{\partial q_{j}}\left[f_{j}(\mathbf{q}) P(\mathbf{q} \mid z)\right]-\sum_{j} \frac{\partial}{\partial q_{j}} \operatorname{Re} \\
& \times \int_{-\infty}^{\infty} \mathrm{d} t g_{j}^{*}(\mathbf{q}, t)\left\langle\prod_{i} \delta\left[q_{i}-q_{i}{ }^{s}(z)\right] \chi(z, t)\right\rangle .
\end{aligned}
$$

To calculate the average in the last term we employ the Furutsu-Novikov formula, ${ }^{26,27}$ which for white noise states that

$$
\left\langle R\left[\chi, \chi^{*}\right] \chi(z, t)\right\rangle=D\left\langle\frac{\delta R\left[\chi, \chi^{*}\right]}{\delta \chi^{*}(\zeta, t)}\right\rangle,
$$

where $R\left[\chi, \chi^{*}\right]$ is an arbitrary functional of white Gaussian noise $\chi$. Applying this formula, we obtain

$$
\begin{aligned}
\left\langle\prod_{i} \delta\left(q_{i}-q_{i}{ }^{s}\right) \chi(z, t)\right\rangle \\
=D\left\langle\frac{\delta}{\delta \chi^{*}(z, t)}\left\{\prod_{i} \delta\left[q_{i}-q_{i}^{s}(z)\right]\right\}\right\rangle \\
=-D \sum_{k} \frac{\partial}{\partial q_{k}}\left\langle\prod_{i} \delta\left[q_{i}-q_{i}^{s}(z)\right] \frac{\delta q_{k}{ }^{s}(z)}{\delta \chi^{*}(z, t)}\right\rangle .
\end{aligned}
$$

Now all that remains is to calculate functional derivative $\delta q_{k}{ }^{s}(z) / \delta \chi^{*}(z, t)$. Using Eq. (18), we can write

$$
\begin{aligned}
q_{k}{ }^{s}(z)= & q_{k}{ }^{s}(0)+\int_{0}^{z} \mathrm{~d} z^{\prime} f_{k}\left(\mathbf{q}^{\mathbf{s}}\right) \\
& +\frac{1}{2} \int_{0}^{z} \mathrm{~d} z^{\prime} \int_{-\infty}^{\infty} \mathrm{d} t^{\prime} g_{k} *\left(\mathbf{q}^{\mathbf{s}}, t^{\prime}\right) \chi\left(z^{\prime}, t^{\prime}\right) \\
& +\frac{1}{2} \int_{0}^{z} \mathrm{~d} z^{\prime} \int_{-\infty}^{\infty} \mathrm{d} t^{\prime} g_{k}\left(\mathbf{q}^{\mathbf{s}}, t^{\prime}\right) \chi^{*}\left(z^{\prime}, t^{\prime}\right) .
\end{aligned}
$$

Now we can calculate the derivative sought as a formal limit:

$$
\frac{\delta q_{k}{ }^{s}(z)}{\delta \chi^{*}(z, t)}=\lim _{\substack{z^{\prime} \rightarrow z \\ t^{\prime} \rightarrow t}} \frac{\delta q_{k}{ }^{s}(z)}{\delta \chi^{*}\left(z^{\prime}, t^{\prime}\right)}=\frac{1}{2} \theta(0) g_{k}\left[\mathbf{q}^{\mathbf{s}}(z), t\right],
$$

where $\theta(0)$ is the value of the Heaviside function at the origin, which is of course an ill-defined quantity. However, putting $\theta(0) \equiv 1 / 2$ will correspond to the assumed Stratonovich interpretation of the Langevin equation because the multiplicative noise term in the Stratonovich framework is symmetrically regularized. Therefore the desired average reads as

$$
\left\langle\prod_{i} \delta\left(q_{i}-q_{i}^{s}\right) \chi(z, t)\right\rangle
$$

$$
=-\frac{D}{4} \sum_{k} \frac{\partial}{\partial q_{k}}\left[g_{k}(\mathbf{q}, t) P(\mathbf{q} \mid z)\right],
$$

and we can finally write the FPE in closed form:

$$
\begin{aligned}
\frac{\partial P(\mathbf{q}, z)}{\partial z}= & -\sum_{j} \frac{\partial}{\partial q_{j}}\left[f_{j}(\mathbf{q}) P(\mathbf{q}, z)\right] \\
& +\frac{D}{4} \sum_{j} \frac{\partial}{\partial q_{j}} \operatorname{Re} \int_{-\infty}^{\infty} \mathrm{d} t g_{j}^{*}(\mathbf{q}, t) \\
& \times \sum_{k} \frac{\partial}{\partial q_{k}}\left[g_{k}(\mathbf{q}, t) P(\mathbf{q}, z)\right] \\
= & -\sum_{j} \frac{\partial}{\partial q_{j}}\left[D_{j}(\mathbf{q}) P(\mathbf{q} \mid z)\right] \\
& +\sum_{j, k} \frac{\partial^{2}}{\partial q_{j} \partial q_{k}}\left[D_{j k}(\mathbf{q}) P(\mathbf{q} \mid z)\right] .
\end{aligned}
$$

Here $D_{j}$ and $D_{j k}$ are the components of the advection vector and of the diffusion matrix, respectively. They are given by the following expressions:

$$
\begin{aligned}
& D_{j}(\mathbf{q})=f_{j}(\mathbf{q})+\frac{D}{4} \sum_{k} \operatorname{Re} \int_{-\infty}^{\infty} \mathrm{d} t g_{k}(\mathbf{q}, t) \frac{\partial g_{j}^{*}(\mathbf{q}, t)}{\partial q_{k}} \\
& D_{j k}(\mathbf{q})=\frac{D}{4} \operatorname{Re} \int_{-\infty}^{\infty} \mathrm{d} t g_{j} *(\mathbf{q}, t) g_{k}(\mathbf{q}, t) .
\end{aligned}
$$

All that is left is to calculate those functions by using definitions (9)-(12). This yields Eq. (24). Note that the FPE [Eq. (A3)] can be written as a conservation law:

$$
\frac{\partial P}{\partial z}=-\sum_{j} \frac{\partial J_{j}}{\partial q_{j}}
$$

where probability current $\mathbf{J}$ is given by

$$
J_{j}=D_{j}(\mathbf{q}) P-\sum_{k} \frac{\partial}{\partial q_{k}}\left[D_{j k}(\mathbf{q}) P\right] .
$$

The approach described here is valid also for the derivation of the Ito FPE. One must bear in mind only that the Ito interpretation requires that the theta function that occurs in the derivation above be put to zero: $\theta(0)$ $=0$. Also, in differentiating Eq. (A1) with respect to $z$ one must remember the chain rule of Ito calculus [Eq. (20)].

\section{ACKNOWLEDGMENTS}

This study was supported by Leverhulme Trust Project F/00250/B (for S. A. Derevyanko and S. K. Turitsyn). The authors are grateful to A. Mecozzi, T. Lakoba, and J. Prilepsky for valuable discussions. 


\section{REFERENCES}

1. J. P. Gordon and H. A. Haus, "Random walk of coherently amplified solitons in optical fiber transmission," Opt. Lett. 11, 665-667 (1986).

2. J. N. Elgin, "Stochastic perturbations of optical solitons," Opt. Lett. 18, 10-12 (1993).

3. A. Mecozzi, J. D. Moores, H. A. Haus, and Y. Lai, "Soliton transmission control," Opt. Lett. 16, 1841-1843 (1991).

4. L. F. Mollenauer, J. P. Gordon, and S. G. Evangelides, "The sliding frequency-guiding filters: an improved form of soliton jitter control," Opt. Lett. 17, 1575-1577 (1992).

5. M. Nakazawa, K. Yamada, H. Kubota, and E. Suzuki, "10 Gbit/s soliton data transmission over one million kilometers," Electron. Lett. 27, 1270-1272 (1991).

6. E. Iannone, F. Matera, A. Mecozzi, and M. Settembre, Nonlinear Optical Communication Networks (Wiley, New York, 1998).

7. H. A. Haus and W. S. Wong, "Solitons in optical communications," Rev. Mod. Phys. 68, 432-444 (1996).

8. A. Mecozzi, "Soliton transmission control by Butterworth filters," Opt. Lett. 20, 1859-1861 (1995).

9. M. Hanna, H. Porte, J. P. Goedgebuer, and W. T. Rhodes, "Soliton optical phase control by use of in-line filters," Opt. Lett. 24, 732-734 (1999).

10. M. Hanna, H. Porte, J. P. Goedgebuer, and W. T. Rhodes, "Experimental investigation of soliton optical phase jitter," IEEE J. Quantum Electron. 36, 1333-1338 (2000).

11. M. Hanna, H. Porte, J. P. Goedgebuer, and W. T. Rhodes, "Performance assesment of DPSK soliton transmission system," Electron. Lett. 37, 644-646 (2001).

12. C. J. McKinstrie and C. Xie, "Phase jitter in single channel soliton systems with constant dispersion," IEEE J. Sel. Top. Quantum Electron. 8, 616-625 (2002).

13. G. Falkovich, I. Kolokolov, V. Lebedev, and S. Turitsyn, "Statistics of soliton-bearing systems with additive noise," Phys. Rev. E 63, 025601(R) (2001).
14. G. Falkovich, I. Kolokolov, V. Lebedev, V. Mezentsev, and S. Turitsyn, "NonGaussian error probability in optical soliton transmission," Physica D 195, 1-28 (2004).

15. C. J. McKinstrie and T. I. Lakoba, "Probability-density function for energy perturbations of isolated optical pulses," Opt. Express 11, 3628-3648 (2003), http:// www.opticsexpress.org.

16. A. Mecozzi, "Probability density functions of the nonlinear phase noise," Opt. Lett. 29, 673-675 (2004).

17. K. P. Ho, "Non-Gaussian statistics of soliton timing jitter induced by amplifier noise," Opt. Lett. 28, 2165-2167 (2003).

18. K. P. Ho, "Phase statistics of the soliton," J. Opt. Soc. Am. B 21, 266-272 (2004).

19. S. A. Derevyanko, S. K. Turitsyn, and D. A. Yakushev, "NonGaussian statistics of an optical soliton in the presence of amplified spontaneous emission," Opt. Lett. 28, 2097-2099 (2003).

20. B. A. Malomed and N. Flytzanis, "Fluctuational distribution function of solitons in the nonlinear Schrödinger system," Phys. Rev. E 48, R5-R8 (1993).

21. D. J. Kaup, "Perturbation theory for solitons in optical fibers," Phys. Rev. A 42, 5689-5694 (1990).

22. H. A. Haus and Y. Lai, "Quantum theory of soliton squeezing: a linearized approach,” J. Opt. Soc. Am. B 7, 386-392 (1990).

23. C. W. Gardiner, Handbook of Stochastical Methods, 2nd ed. (Springer-Verlag, Berlin, 1996).

24. P. E. Kloeden and E. Platen, The Numerical Solution of Stochastic Differential Equations (Springer-Verlag, Berlin, 1992).

25. P. Gaspard, "Trace formula for noisy flows," J. Stat. Phys. 106, 57-96 (2002)

26. K. Furutsu, "On the statistical theory of electromagnetic waves in a fluctuating media," J. Res. Natl. Bur. Stand. Sect. D 67, 303-323 (1963).

27. Y. Novikov, "Functionals and the random force method in turbulence theory," Sov. Phys. JETP 20, 1290-1294 (1965). 